\title{
Stimuli-Responsive Hydrogels Synthesis using Free Radical and RAFT Polymerization
}

\author{
Miguel A. D. Gonçalves, ${ }^{1}$ Virgínia D. Pinto, ${ }^{1}$ Rita A. S. Costa,${ }^{1}$ Rolando C. S. Dias,${ }^{* 1}$ \\ Julio C. Hernándes-Ortiz, ${ }^{2}$ Mário Rui P. F. N. Costa ${ }^{2}$
}

Summary: Temperature and $\mathrm{pH}$ stimuli-responsive hydrogel particles were synthesized using inverse-suspension polymerization in batch stirred reactor. Different water soluble co-monomers were present in the initial mixture (e.g. $\mathrm{N}$-isopropylacrylamide and acrylic acid) as well as crosslinkers with different functionalities. Different operating conditions such as polymerization temperature, monomers dilution, neutralization and the initial ratios of co-monomers and monomers/crosslinker were also tried. Hydrogel particles were produced considering classical free-radical polymerization (FRP) and also RAFT polymerization. Commercially available RAFT agents 4-cyano-4-phenylcarbonothioylthio-pentanoic acid (CPA), 2-(dodecylthiocarbonothioylthio)-2-methylpropionic acid (DDMAT) and cyanomethyl dodecyl trithiocarbonate (CDT) were alternatively used. Sampling at different polymerization times allowed the study of the kinetics of polymerization through the analysis by SEC of the soluble phase. A tetra-detector array with simultaneous detection of refractive index, light scattering, intrinsic viscosity and ultra-violet signals was used in these studies. Usefulness of in-line FTIR-ATR monitoring to study the building process of such networks was also assessed. The performance of hydrogel beads was studied through drug delivery tests triggered by changes in the environmental temperature and $\mathrm{pH}$. This research aims to contribute for the elucidation of the connection between the synthesis conditions, molecular architecture and properties/performance of such advanced materials.

Keywords: crosslinking; hydrogels; RAFT; stimuli-responsive polymers

\section{Introduction}

Hydrogels have been extensively studied in last decades due to their potential new applications in biotechnology and biomedicine $^{[1,2]}$. Researches on this field are specially focused on the so called smart hydrogels (or stimuli responsive hydrogels) which microscopic properties are sensitive to changes triggered by the environmental conditions. Generically speaking, formation of soluble networks and gels has been

\footnotetext{
${ }^{1}$ LSRE-Instituto Politécnico de Bragança, Quinta de Santa Apolónia, 5300, Bragança, Portugal E-mail: rdias@ipb.pt

2 LSRE-Faculdade de Engenharia da, Universidade do Porto, Rua Roberto Frias s/n, 4200-465, Porto, Portugal
}

experimentally and theoretically studied since the beginning of polymer science. With systems involving vinyl/multivinyl monomers, classical free radical polymerization (FRP) mechanisms were mainly considered in these studies. A new importance was given to this subject with the advent of controlled radical polymerization (CRP). New studies in this research area were driven by the possibility of improvement of networks and gels properties as result of higher structural homogeneity. In fact, in the last years, the three main CRP techniques (ATRP ${ }^{[3-9]}, \mathrm{NMRP}^{[8-15]}$, and RAFT $^{[16-19]}$ ) were exploited aiming the production of advanced polymer networks belonging to different classes (e.g. organic or water compatible materials). 
RAFT polymerization can be used in a broad range of operation conditions and with different monomer classes, including water compatible monomers. These advantages are explored in this work considering the inverse-suspension synthesis of different classes of stimuli-responsive hydrogels particles and using three different commercially available RAFT agents. FRP synthesis of the same materials was also performed in order to highlight the differences between the two processes (namely in the observed kinetics of polymerization). The use of different polymer characterization methods (such as SEC with tetradetection and in-line FTIR-ATR monitoring) to study the formation of such networks is illustrated. The usefulness of such techniques to describe the crosslinking process is discussed. Final applications of the produced materials are also tested through drug delivery studies. Experimental results here reported aims to contribute to the sought linking between the production conditions of advanced materials and their structure/properties. Development of tools helping in the synthesis of tailored materials is the ultimate goal of this research.

\section{Experimental}

\section{Materials}

N-isopropylacrylamide (NIPA) of $99 \%$ purity, N,N-dimethylacrylamide (DMA) of $99 \%$ purity stabilized with $500 \mathrm{ppm}$ monomethyl ether hydroquinone (MEHQ), 2-(dimethylamino)ethyl methacrylate (DMAEMA) of $98 \%$ purity stabilized with $700-1000 \mathrm{ppm}$ MEHQ, acrylic acid (AA) of $99 \%$ purity stabilized with 180-200 ppm MEHQ, methacrylic acid (MAA) of $99 \%$ purity stabilized with $250 \mathrm{ppm}$ MEHQ, N,N'-methylenebisacrylamide (MBAm) of $99 \%$ purity, ethylene glycol dimethacrylate (EGDMA) of $98 \%$ purity stabilized with 90-110 ppm MEHQ, trimethylolpropane triacrylate (TMPTA) stabilized with $100 \mathrm{ppm}$ methylethylhydroquinone, 1,1,2,2 - tetraallyloxyethane (TAO), hydroquinone of $99 \%$ purity, AIBN of $98 \%$ purity, 2,2'-azobis(2-methylproprionamidine) dihydrochloride (V50) of $98 \%$ purity, ammonium persulfate (APS) of $98 \%$ purity and $\mathrm{N}, \mathrm{N}, \mathrm{N}^{\prime}, \mathrm{N}^{\prime}$-tetramethylethylenediamine (TEMED) of $99 \%$ purity were purchased from Sigma Aldrich and used as received. The commercially available RAFT agents 2-(dodecylthiocarbonothioylthio)-2-methylpropionic acid (DDMAT) of $98 \%$ purity, 4-cyano-4-(phenylcarbonothioylthio)pentanoic acid (CPA) of $97 \%$ purity and cyanomethyl dodecyl trithiocarbonate (CDT) of $98 \%$ purity were also purchased from Sigma Aldrich and used as received. Dimethylformamide (DMF) of $99.5 \%$ purity (Fisher Scientific), tetrahydrofuran (THF) of $99 \%$ purity (Fisher Scientific) and cyclohexane of $99 \%$ purity (Sigma Aldrich) were also used as received. A current grade of liquid paraffin was used when needed. Caffeine of $98.5 \%$ purity, ibuprofen of $99 \%$ purity, 5-fluorouracil $(5 \mathrm{Fu})$ of $98.5 \%$ purity and isonicotinic acid hydrazide (isoniazid) of $99 \%$ purity were purchased from Acros Organics and used as model chemicals in the drug release tests performed. Ibuprofen was also transformed in its sodium salt in order to increase the solubility of the drug in the aqueous solutions considered.

\section{Polymerization Runs}

Hydrogels were synthesized in batch reactor using the inverse suspension process. Polymerizations were performed at $200 \mathrm{~mL}$ total volume scale with stable suspension formation using a volumetric ratio aqueous/ organic phases $=1 / 5,1 \%(\mathrm{w} / \mathrm{w})$ of surfactant (span 80) in the continuous phase and agitation speed at $300 \mathrm{rpm}$. Gel production at isothermal conditions and keeping a good stirring of the reaction vessel was possible using these conditions. When applicable, reactants were previously bubbled with argon, that was also sweep in the reaction medium during the polymerizations in order to prevent inhibition by oxygen. At prescribed polymerization times, reaction samples were collected from the reactor, quenched at low temperature in a solution containing hydroquinone to stop 
the reactions, and afterwards prepared for injection of the soluble polymer in the SEC system $^{[20]}$. Morphology of the final gel beads, after products purification, was also characterized by SEM. Micro appearance of the produced materials is illustrated in Figure 1.

Tables 1-3 describe the details of a set of experiments performed in this research. Different polymerizations were performed combining different water compatible vinyl monomers and crosslinkers. Initiation system was also changed along the experimental program. Conventional (FRP) and RAFT mechanisms were used. Besides these conditions, the following main para-

(a)

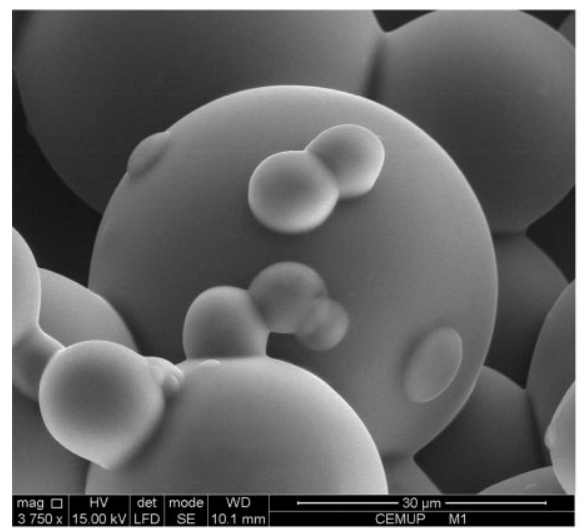

(c)

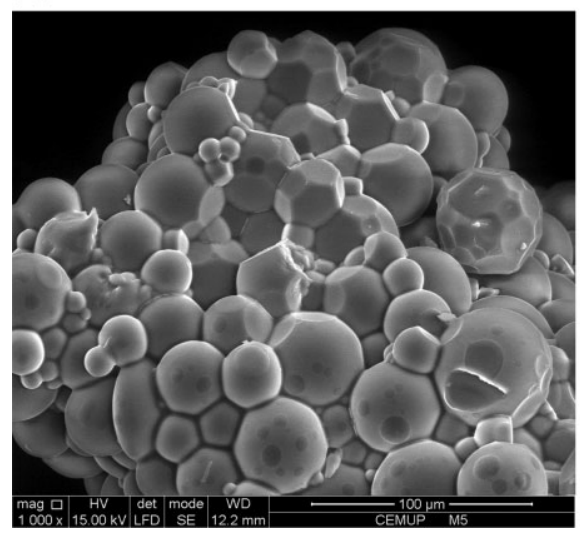

meters describe the set of polymerization runs:

- Initial mole fraction of co-vinyl monomer $\left(\mathrm{M}_{1}\right)$ in the binary mixture of $\mathrm{M}_{1}+\mathrm{M}_{2}$ $\left(Y_{M 1}\right)$.

- Initial mole ratio between initiator and monomers $\left(Y_{I}\right)$.

- Initial mole fraction of crosslinker in the total monomer mixture $\left(Y_{C L}\right)$.

- Initial mass fraction of the monomers in the dispersed phase $\left(Y_{m}\right)$.

- Initial mole ratio between RAFT agent and initiator $\left(Y_{I}^{R A F T}\right)$.

Slightly different reaction conditions were used in the experimental runs 1 (b)

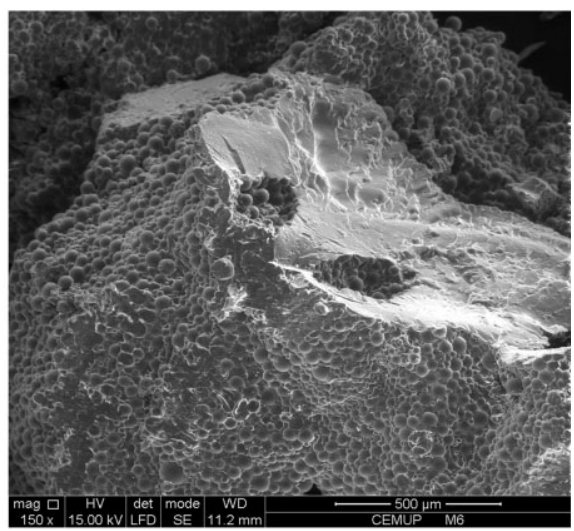

(d)

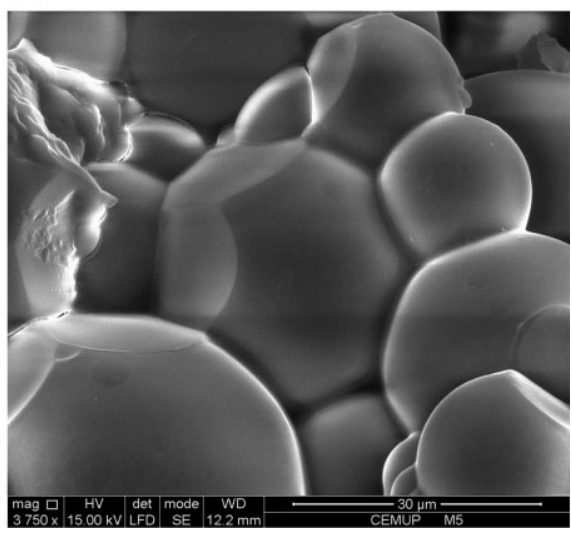

\section{Figure 1.}

SEM micrographs of some hydrogel beads synthesized in this work using the inverse-suspension technique. (a) AA/MBAm hydrogel. (b) NIPA/MBAm hydrogel. (c) and (d) NIPA/AA/MBAm hydrogel. In some cases, formation of fused material was observed due to the post-treatment of the products (precipitation/drying). 
Table 1.

A set of polymerization runs performed in the inverse-suspension FRP synthesis of $\mathrm{pH} / \mathrm{Temperature}$ responsive hydrogels. Water was used as solvent in the dispersed phase and cyclohexane was considered as continuum medium. Polymerizations at $20^{\circ} \mathrm{C}$.

\begin{tabular}{lllllrrrr}
\hline Run & $\mathrm{M}_{1}$ & $\mathrm{M}_{2}$ & $\mathrm{CL}$ & $\mathrm{I}$ & $Y_{M_{1}}(\%)$ & $Y_{C L}(\%)$ & $Y_{l}(\%)$ & $Y_{m}(\%)$ \\
\hline 1 & NIPA & - & MBAm & APS & 100 & 1 & 0.25 & 10 \\
2 & NIPA & AA & MBAm & APS & 50 & 1 & 0.25 & 14.5 \\
3 & NIPA & MAA & MBAm & APS & 67 & 1 & 0.25 & 13.8 \\
4 & NIPA & AA & MBAm & APS & 88 & 1 & 0.26 & 11.1 \\
5 & NIPA & - & TAO & APS & 100 & 1 & 0.25 & 10 \\
6 & NIPA & - & TMPTA & APS & 100 & 1 & 0.25 & 10 \\
7 & NIPA & - & MBAm & APS & 100 & 2 & 0.25 & 10 \\
\hline
\end{tabular}

Table 2.

A set of polymerization runs performed in the inverse-suspension RAFT synthesis of water compatible polymers and hydrogels. DMF was used as solvent in the dispersed phase and liquid paraffin was considered as continuum medium. Polymerizations at $70^{\circ} \mathrm{C}$. DDMAT was used as RAFT agent.

\begin{tabular}{llcccccccc}
\hline Run & $\mathrm{M}_{1}$ & $\mathrm{M}_{2}$ & $\mathrm{CL}$ & $\mathrm{I}$ & $Y_{M 1}(\%)$ & $Y_{C L}(\%)$ & $Y_{l}(\%)$ & $Y_{m}(\%)$ & $Y_{I}^{\text {RAFT }}$ \\
\hline 1 & NIPA & - & - & AIBN & 100 & 0 & 0.24 & 25.6 & 4.18 \\
2 & AA & - & - & AIBN & 100 & 0 & 0.23 & 17.9 & 4.42 \\
3 & DMA & - & - & AIBN & 100 & 0 & 0.06 & 33.2 & 5.03 \\
4 & MAA & - & - & AIBN & 100 & 0 & 0.05 & 33.6 & 5.00 \\
5 & AA & - & - & AIBN & 100 & 0 & 0.03 & 40.4 & 8.69 \\
6 & AA & - & MBAm & AIBN & 100 & 1 & 0.04 & 40.3 & 8.54 \\
7 & NIPA & - & MBAm & AIBN & 100 & 1 & 0.04 & 30.0 & 8.96 \\
8 & NIPA & AA & MBAm & AIBN & 90 & 1 & 0.04 & 31.0 & 9.00 \\
\hline
\end{tabular}

and 3 described in Table 3, aiming the improvement of the in-line FTIR-ATR measurements. Liquid paraffin was used to minimize the IR absorption of the continuum medium and solvent (e.g. water or DMF) was not used in the polymerization phase to eliminate the usual strong influence of these compounds on the IR spectra of monomers and produced polymers. Liquid paraffin was also considered in order to promote a low thermodynamic affinity with monomers and produced gels. Precipitation of the products in the particulate form along the polymerization was thus observed and good stirring conditions, as well as, good heat dissipation could be maintained during the reactions. After purification, powder gels were obtained as final products. Effect of other synthesis conditions on the crosslinking process, such as, presence of solvent and the nature of monomers and RAFT agents, was also

\section{Table 3.}

A set of polymerization runs performed in the synthesis of water compatible polymers and hydrogels considering liquid paraffin as continuum medium. EGDMA was used as crosslinker in runs 1-3 and MBAm in runs 4-5. Polymerizations at $60^{\circ} \mathrm{C}$ with exception of run $4\left(50^{\circ} \mathrm{C}\right)$. Water as solvent in runs 2 and 4 and DMF in run 5. RAFT agents used: CPA in runs $3-4$ and CDT in run 5.

\begin{tabular}{lcccccccc}
\hline Run & $M_{1}$ & $M_{2}$ & $\mathrm{I}$ & $Y_{M 1}(\%)$ & $Y_{C L}(\%)$ & $Y_{l}(\%)$ & $Y_{M 1}(\%)$ & $Y_{I}^{\text {RAFT }}$ \\
\hline 1 & DMAEMA & - & AIBN & 100 & 4.55 & 0.5 & 100 & 0 \\
2 & DMAEMA & - & $V_{50}$ & 100 & 4.88 & 0.3 & 73 & 0 \\
3 & DMAEMA & - & AIBN & 100 & 4.76 & 0.5 & 100 & 1.98 \\
4 & DMAEMA & MAA & $V_{50}$ & 35 & 2 & 0.14 & 50 & 1.94 \\
5 & NIPA & AA & AIBN & 88 & 1 & 0.36 & 22 & 1.99 \\
\hline
\end{tabular}


assessed in this set of polymerizations as reported with the remaining experimental runs of Table 3.

\section{Product Analysis by SEC with a Tetra Detector Array}

The SEC apparatus used is composed of a Viscotek GPCmax VE 2001 integrated solvent and sample delivery module coupled to a tetra detector array including refractive index (RI), light scattering (LS), viscosity (IV-DP) and ultraviolet (UV) detection. Analysis were performed directly in aqueous eluents ( $\mathrm{pH}$ of the eluent was changed according the polymer analysed) and using typically a flow-rate of $0.5 \mathrm{~mL} /$ min. Temperature of the analysis (in the range 30 to $50^{\circ} \mathrm{C}$ ) was also changed considering the different conformations of the polymers in aqueous solutions (e.g. collapsing of NIPA based materials at around $37^{\circ} \mathrm{C}$ ). A train of 3 SEC columns (Viscotek A2000 + Viscotek A3000 + Viscotek A6000) was considered to fractionate the polymers by size (different configurations were also used in order to not exceed the recommended maximum columns pressures). Simultaneous measurement of RI, LS and intrinsic viscosity signals yield absolute molecular weight, branching factors, hydrodynamic radius and radius of gyration of the soluble phase. Monomer conversion was also estimated through the measurement of the monomers peak areas in these chromatograms. Typical results concerning the analysis of water soluble polymers with this apparatus are illustrated in Figure 2. Simultaneous detection of three signals (RI, LS and Intrinsic Viscosity) allows the detailed characterization of the molecular architecture of the soluble phase and the observation of the influence of the production conditions (e.g. comparison FRP/RAFT synthesis) on the dynamics of crosslinking. Light scattering measurements proved to be especially important because show the possibility of occurrence of non-ideal RAFT polymerization, as below described. In this research, UV detection was used to carry out drug release studies with the synthesized hydrogels, as also below described.

\section{In-Line FTIR-ATR Measurements}

An Attenuated Total Reflection (ATR) immersion probe, coupled to a Fourier Transform Infra-Red (FTIR) spectrophotometer (which technical features were described elsewhere ${ }^{[7]}$ ) was used to perform the in-line monitoring of polymerization runs, aiming the measurement of the building process of the networks. Typical results obtained with the in-line FTIR-ATR monitoring of hydrogel formation are illustrated in Figure 3. These runs were designed in order to have optimum
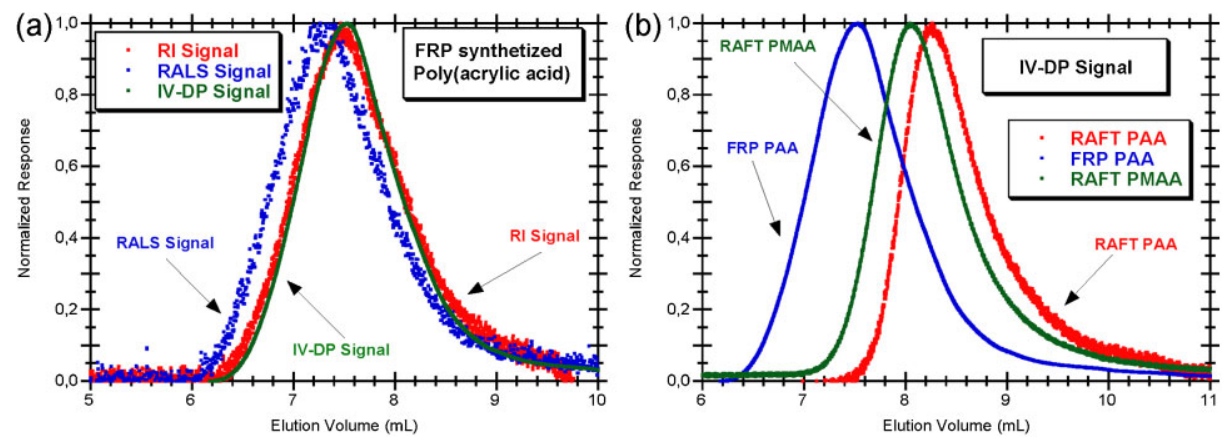

Figure 2.

(a) Refractive Index (RI), Right Angle Light Scattering (RALS) and Intrinsic Viscosity - Differential Pressure (IV-DP) signals simultaneously observed in the SEC analysis of a water soluble PAA sample. (b) IV-DP signals observed in the SEC analysis of different water soluble polymers synthesized in this research, highlighting the influence of operation conditions (e.g. FRP/RAFT) on the products molecular structure and properties. 
(a)

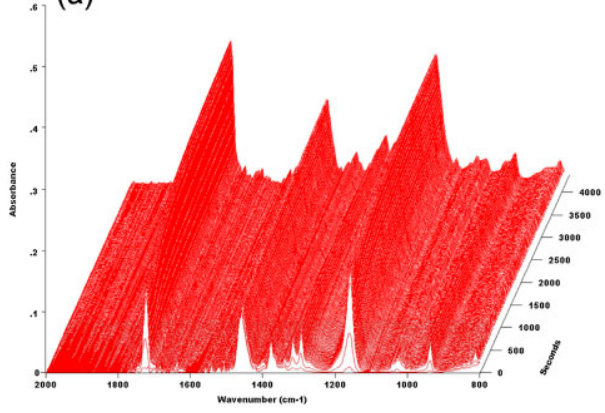

(b)

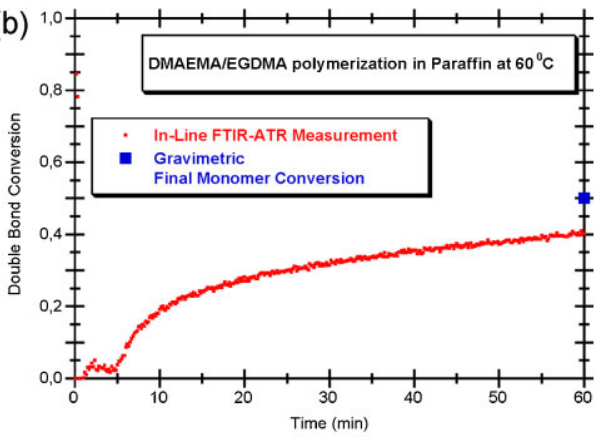

Figure 3.

(a) In-line FTIR-ATR spectra observed during DMAEMA/EGDMA FRP polymerization (run 1 in Table 3). Absorption peak at around $935 \mathrm{~cm}^{-1}$ was considered to estimate the double bonds conversion, using also the peak at around $1720 \mathrm{~cm}^{-1}$ as internal reference. (b) FTIR-ATR estimated dynamics of monomer conversion for DMAEMA/ EGDMA polymerization considering FRP polymerization (run 1 in Table 3). Similar measurements were performed with runs 2 and 3 in Table 3 but even lower monomer conversions were observed in these experiments (almost negligible after 8 hours of polymerization in run 3 ).

conditions for FTIR measurements (minimizing possible interferences with monomers/polymer spectra) but a limited information concerning the crosslinking process was obtained. Coating of the probe at relative low monomer conversion (e.g. around $40 \%$ as recently reported in other studies $^{[22]}$ ) is a possible shortcoming of these in-line measurements. Off-line FTIR analysis of previously isolated network samples collected at different polymerization times seems to lead to a better description of the crosslinking process, namely concerning pendant double bonds reactivity ${ }^{[23]}$. Crosslinker amount used in hydrogels preparation is very low (a few percent) and even with off-line FTIR monitoring the study of the crosslinking
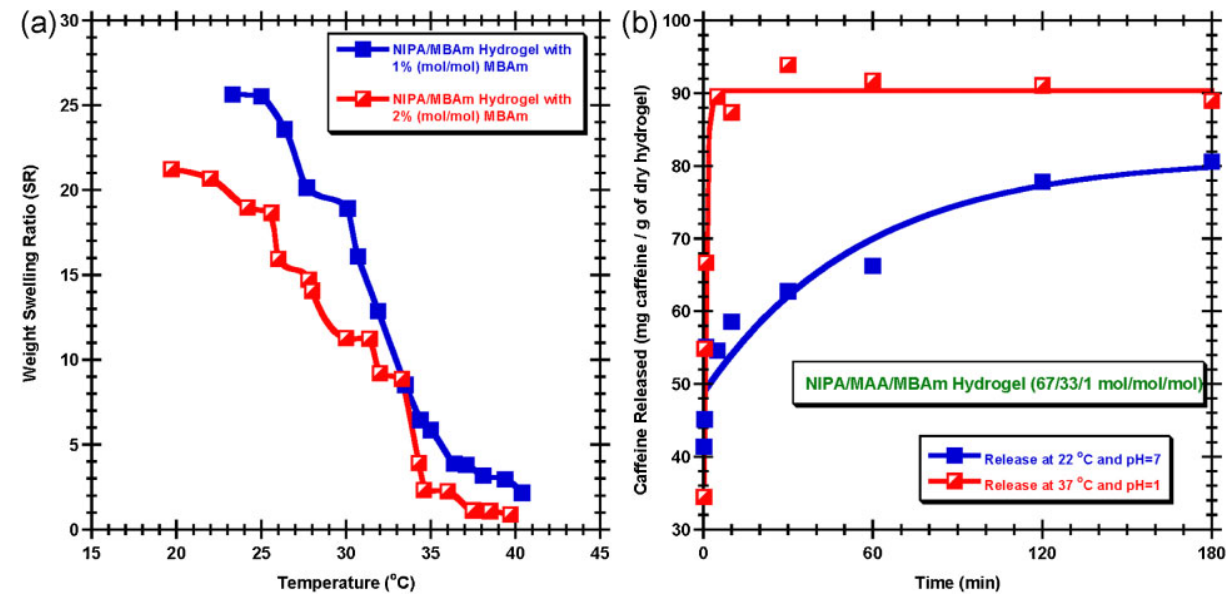

Figure 4.

(a) Measured equilibrium weight swelling ratio of NIPA/MBAm hydrogels in aqueous solutions at different temperatures illustrating networks sensitivity to changes in this parameter. (b) Dynamics of caffeine release from a $\mathrm{pH} /$ temperature sensitive synthesized hydrogel (NIPA/MAA/MBAm) measured by UV detection at $270 \mathrm{~nm}$. Two different surrounding water solutions were considered: $\mathrm{pH}=1 / \mathrm{T}=37^{\circ} \mathrm{C}$ (collapsed particles) and $\mathrm{pH}=7 / \mathrm{T}=22^{\circ} \mathrm{C}$ (swollen particles). Drug loading was performed by swelling the hydrogel beads in caffeine aqueous solution during $48 \mathrm{hr}$. 
process is a difficult task. ${ }^{13} \mathrm{C}$ labelling of the crosslinker (as before performed with trimethylolpropane triacrylate (TMPTA) in the framework of network formation in superabsorbent gels ${ }^{[24]}$ ) should be a better option in this context.

\section{Swelling Ratio Sensitivity Measurements}

Synthesized hydrogel particles, after isolation, were tested in order to assess their sensitivity to stimulations triggered by changes in the surrounding media. In particular, was measured the variation of the hydrogels weight swelling ratio, in water solutions, at different temperature and/ or $\mathrm{pH}$ values. Stimulation of the networks by changes in these parameters was thus observed. Typical results obtained are presented in Figure 4 (a) where sensitivity of NIPA/MBAm hydrogels to temperature changes is used as illustration example.

\section{Drug Release Testing}

Performance of the produced hydrogels was also assessed considering drug delivery applications. Different model drugs (caffeine, 5-fluorouracil, isoniazid and ibuprofen) were considered in these studies that were also carried out pouring pre-incubated network particles in aqueous solutions at different conditions (changing $\mathrm{pH} /$ temperature). Drug release was measured by UV detection in aqueous samples collected at different elapsed times. Typical results are presented in Figure 4 (b) using the caffeine release from a NIPA/MAA/MBAm hydrogel at different $\mathrm{pH} /$ temperature conditions as illustration example. In spite of the complexities associated with the mathematical modelling of drug delivery ${ }^{[1,2]}$, a good agreement is observed fitting the experimental data to exponential rise laws (effect of different stimulations on the drug release profiles observed is also here highlighted).

Applications of the different classes of "smart" hydrogels here studied is further enhanced in Figures 5 and 6. In Figure 5 (a) is presented the measured equilibrium weight swelling ratio of anionic (AA based) and cationic (DMAEMA based) hydrogels in aqueous solutions at different $\mathrm{pH}$ values.
These results illustrate the networks sensitivity to changes in this parameter. Note that inverse effect of the $\mathrm{pH}$ on the swelling ratio of these hydrogels can be explored to trigger different macroscopic effects, as for instance the transition between shrunk to swollen networks by changing the $\mathrm{pH}$ from 1 to 8 (e.g. resembling the stomach/ intestine $\mathrm{pH}$ change in human body) with AA hydrogels and the opposite with DMAEMA hydrogels. In Figure 5 (b) is showed the comparison for the change of the equilibrium weight swelling ratio with $\mathrm{pH}$ considering FRP and RAFT synthesized AA hydrogels. These results illustrate the high effect of the synthesis technique used on the swelling properties of the hydrogels. In fact, the primary chain length of the networks is strongly affected when FRP is replaced by RAFT. This effect can eventually be used to tune the swelling properties of the hydrogels (e.g. designing the initial ratios between monomers/RAFT agent/initiator). It is worth to note that measurements presented in Figures 5 (a) and (b) were obtained using buffer aqueous solutions at different $\mathrm{pH}$ values. These buffer solutions were prepared using the proper amounts of $\mathrm{HCl}, \mathrm{NaOH}, \mathrm{KCl}$, KHP (potassium hydrogen phthalate $\mathrm{C}_{8} \mathrm{H}_{5} \mathrm{KO}_{4}$ ), $\mathrm{KH}_{2} \mathrm{PO}_{4}$ (potassium dihydrogen phosphate), $\mathrm{Na}_{2} \mathrm{~B}_{4} \mathrm{O}_{7} 10 \mathrm{H}_{2} \mathrm{O}$ (borax) and $\mathrm{Na}_{2} \mathrm{HPO}_{4}$ (disodium hydrogen phosphate). Nevertheless, the swelling ratio of hydrogels is also strongly dependent on the ionic strength and size of the ions and counterions present in the used aqueous solutions. Accordingly, a different dependence of the hydrogels swelling ratio on $\mathrm{pH}$ changes can be observed if other aqueous solutions at the same $\mathrm{pH}$ values are considered (e.g. changing the used salts and/or using just $\mathrm{HCl}$ and $\mathrm{NaOH}$ to prepare the aqueous solutions with the desired $\mathrm{pH}$ values).

Measured dynamics of release of different drugs from cationic and anionic hydrogels is illustrated in Figure 6. In Figure 6 (a) and (b) is showed the dynamics of release of 5-fluorouracil from DMAEMA based (cationic) and AA based (anionic) hydrogels, 

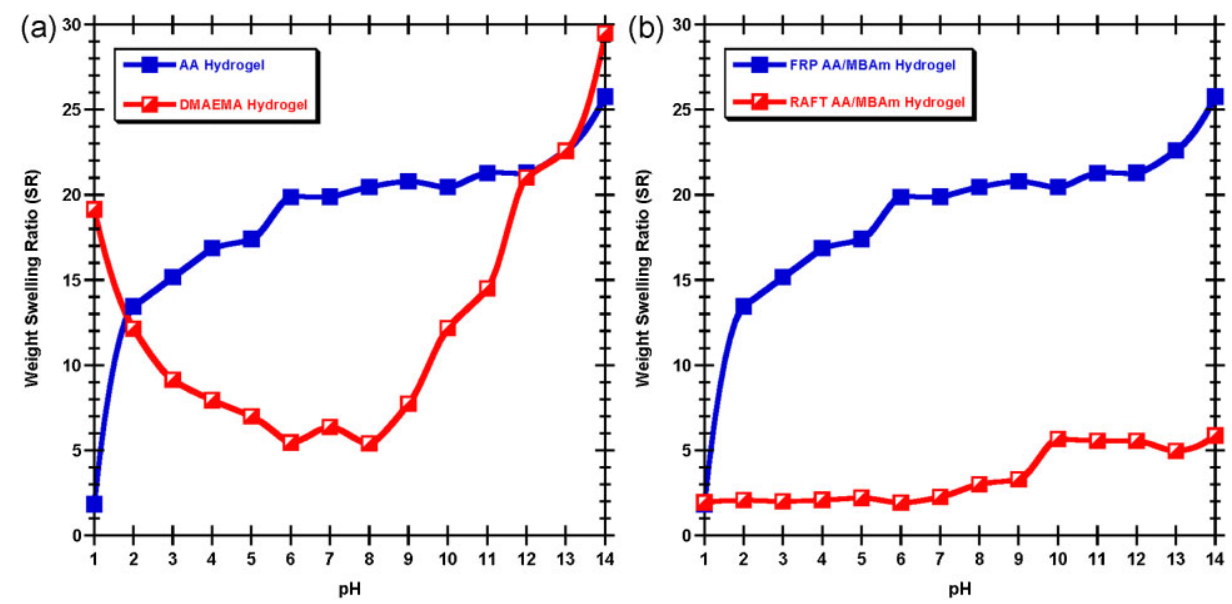

\section{Figure 5.}

(a) Measured equilibrium weight swelling ratio of anionic (AA based) and cationic (DMAEMA based) hydrogels in aqueous solutions at different $\mathrm{pH}$ values illustrating networks sensitivity to changes in this parameter. Inverse effect of the $\mathrm{pH}$ on the swelling ratio of these hydrogels can be explored to trigger different macroscopic effects (e.g. shrunk to swollen networks by changing the $\mathrm{pH}$ from 1 to 8 with AA hydrogels and the opposite with DMAEMA hydrogels). (b) Comparison of the change of the equilibrium weight swelling ratio with pH for FRP and RAFT synthesized AA hydrogels. High effect of the synthesis technique used on this parameter is observed. The primary chain length of the networks is strongly affected when FRP is replaced by RAFT which can eventually be used to tune the swelling properties of the hydrogels. Note that results presented in both Figures ((a) and (b)) were obtained using buffer aqueous solutions at different $\mathrm{pH}$ values. The swelling ratio of hydrogels is also strongly dependent on the ionic strength and size of the ions/counterions present in the solutions. A different dependence of SR on $\mathrm{pH}$ can be observed if other aqueous solutions at the same $\mathrm{pH}$ values are considered (e.g. changing the used salts).

respectively. In both cases, the release of the drug was measured in acidic $(\mathrm{pH}=1)$ and alkaline $(\mathrm{pH}=10)$ aqueous solutions. In spite of the differences between the two hydrogels, slightly higher steady state release of the drug was always observed with the alkaline environment. Note that some other complex effects such as hydrogel/drug interaction ${ }^{[1,2]}$ (e.g. see discussions about diffusion and chemically controlled delivery systems in chapter 11 of $\mathrm{ref}^{[1]}$.) should be take into account when drug release studies are performed, as for instance formation of complexes between drugs and polymer networks. These issues also have a strong effect on the amount of a specific drug that is possible to load in a hydrogel. The effect of the combination between specific drugs and hydrogels is illustrated in Figure 6 (c) where the dynamics of release of ibuprofen from DMAEMA and AA polymer networks, both placed in aqueous solution at $\mathrm{pH}=10$, is showed. Comparison of the dynamics of release of 5-fluorouracil from FRP and RAFT synthesized $\mathrm{pH}$ sensitive hydrogels is illustrated in Figure 6 (d). Amount of drug released is in this case expressed as the fraction of drug loaded in the hydrogel that is transferred to the aqueous solution. Note that much more lower release fractions were observed (both at $\mathrm{pH}=1$ and 10) when RAFT hydrogels were considered. These results should be a consequence of the different molecular architectures associated with FRP and RAFT networks (affecting namely their swelling ratio, as showed in Figure 5 (b)) and highlights the relation between structure and end use properties of these materials.

\section{Results and Discussion}

Reversible addition-fragmentation chain transfer polymerization is probably the 

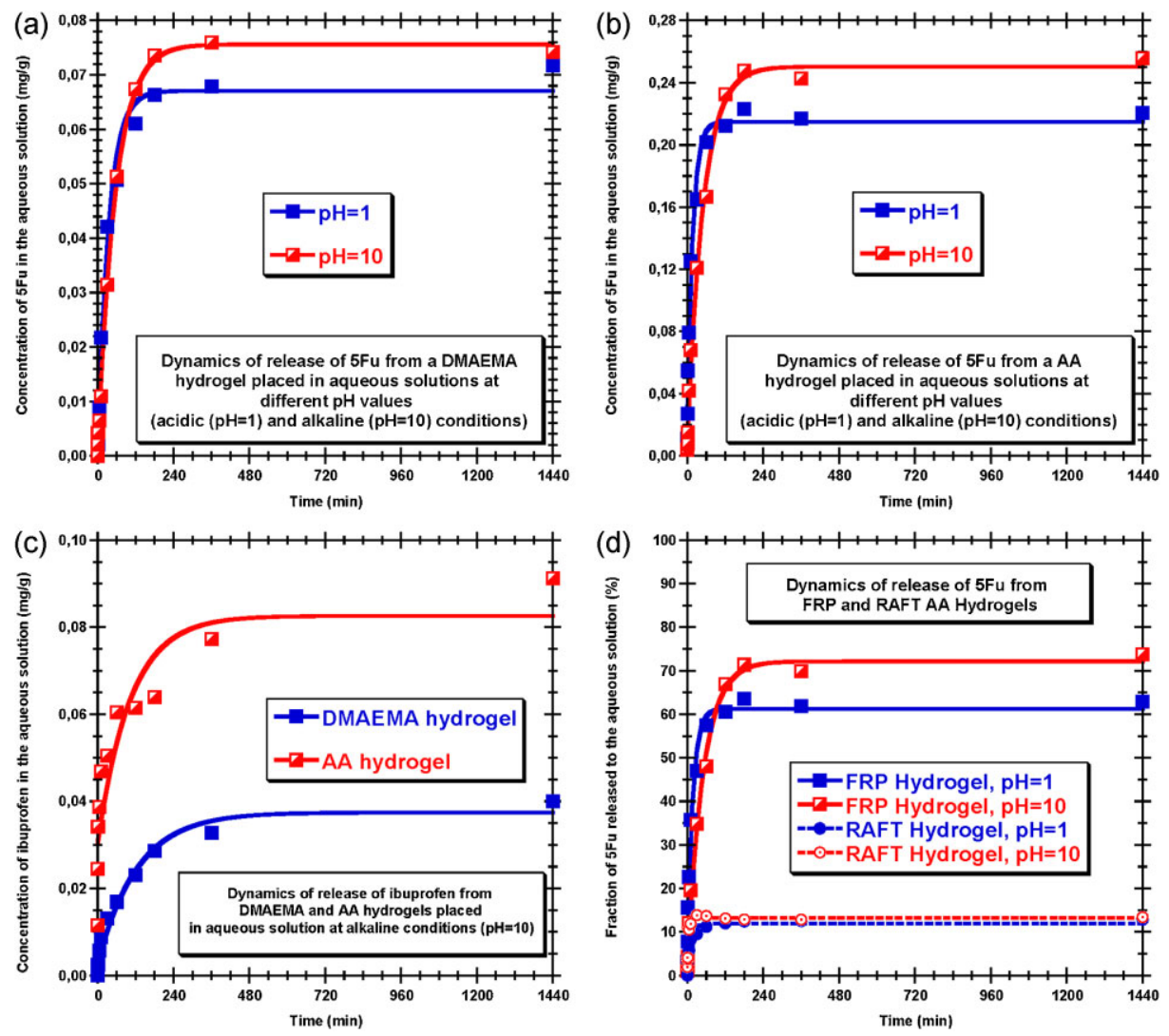

\section{Figure 6.}

(a) Dynamics of release of 5 -fluorouracil from a pH sensitive hydrogel (cationic hydrogel based on DMAEMA) measured by UV detection at $270 \mathrm{~nm}$. (b) Dynamics of release of 5 -fluorouracil from a pH sensitive hydrogel (anionic hydrogel based on AA) measured by UV detection at $270 \mathrm{~nm}$. (c) Comparison of the dynamics of release of ibuprofen from cationic (DMAEMA based) and anionic (AA based) hydrogels, both placed in aqueous solution at $\mathrm{pH}=10$ (release measured by UV detection at $223 \mathrm{~nm}$ ). (d) Dynamics of release of 5 -fluorouracil from FRP and RAFT synthesized pH sensitive hydrogels illustrating the effect of the molecular architecture of the networks on their performance. Amount of drug released is here expressed as the fraction of drug loaded in the hydrogel that is transferred to the aqueous solution (release measured by UV detection at $270 \mathrm{~nm}$ ). In all cases presented in this Figure, drugs loadings were performed by swelling the hydrogels in 5 -fluorouracil or ibuprofen aqueous solutions during $48 \mathrm{hr}$.

most versatile CRP technique allowing the polymerization of different classes of monomers. Nevertheless, the degree of control of polymerization that is attained with RAFT is strongly dependent on the reaction conditions used. Specific combination between monomer, RAFT agent, initiator and solvent used in the polymerization is a central issue to obtain tailored products with RAFT polymerization. Temperature and initial proportions monomer/ RAFT agent/initiator/solvent also have a huge effect on the kinetics of formation and on the control of the molar masses of RAFT polymers. Some other issues arise when RAFT is directly performed in water, namely the low solubility of most RAFT agents in pure water (forcing the use organic co-solvents) and their potential hydrolysis ( $\mathrm{pH}$ dependent) with loss of control on the polymerization process (see ${ }^{[25]}$ and references therein). When aqueous dispersed systems are considered (e.g. the industrially important emulsion/ 
miniemulsion/suspension processes and their inverse counterparts) some other aspects like the transport of reactants (monomers, initiators, RAFT agents) between organic and aqueous phases become also of crucial importance. In this context, the RAFT inverse miniemulsion of acrylamide and acrylic acid were recently reported $^{[26,27]}$ and the effect of $\mathrm{pH}$ on the hydrolysis of the RAFT agent and polymerization in the continuous phase (eventually in the absence of RAFT agent) were identified as phenomena potentially involved in some loss of control observed with particular conditions. A secondary peak was observed in the RI curve (see discussion below in the context of the results here presented) which was attributed to different polymer populations formed in both phases (aqueous and organic). These aspects get an additional importance in the framework of RAFT dispersed systems that has been very recently explored to produce amphiphilic copolymers and nanoparticles/nano-objects with different morphologies (e.g. spheres, fibers, vesicles $)^{[28-30]}$. Bellow are discussed some of our findings involving probably related mechanisms that are present in the RAFT inverse suspension formation of hydrogels or their linear counterparts.

Very fast reactions are generally involved in the FRP synthesis of hydrogels, as before showed with different classes of monomers ${ }^{[20,21]}$. Fast gelation is observed with a few percent of crosslinker agent and parameters such as polymerization temperature, monomer concentration and neutralization have a very strong influence on the dynamics of gelation (see results before presented in refs $\left.{ }^{[20,21]}\right)$. Similar behaviour was observed in the FRP polymerization runs detailed in Table 1 , even considering a low polymerization temperature $\left(\mathrm{T}=20^{\circ} \mathrm{C}\right)$.

It is known that more amenable kinetics of polymerization can be achieved replacing FRP by RAFT polymerization. Design of operation conditions, namely the initial proportions between initiator/RAFT agent/ monomer, can be used to manipulate reaction rates and also to design the degree of polymerization. With network formation, these parameters can be used to try the manipulation of the primary chain length (thus affecting gelation) and the minimization of intramolecular reactions (cyclizations) leading to the decrease of crosslinking efficiency.

If direct aqueous polymerization is intended, as in many cases involving hydrogels, a major problem to be faced with RAFT polymerization is the low water solubility of usual RAFT agents. This issue applies to DDMAT and therefore the aqueous RAFT polymerization is not possible. This problem can be circumvented using a different solvent, such as DMF, as described in Table 2. Under these conditions reasonable reaction rates were
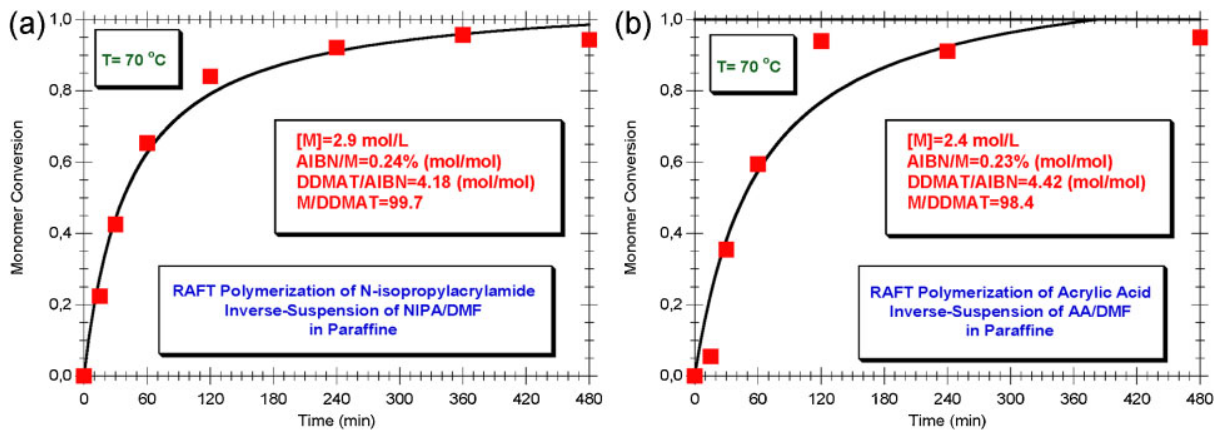

Figure 7.

Examples of observed dynamics of monomer conversion in different runs concerning the inverse-suspension RAFT synthesis of water compatible polymers and hydrogels (see Table 2). (a): NIPA RAFT polymerization with $Y_{1}^{\text {RAFT }}=4.18$ (run 1). (b): AA RAFT polymerization with $Y_{1}^{\text {RAFT }}=4.42$ (run 2). 

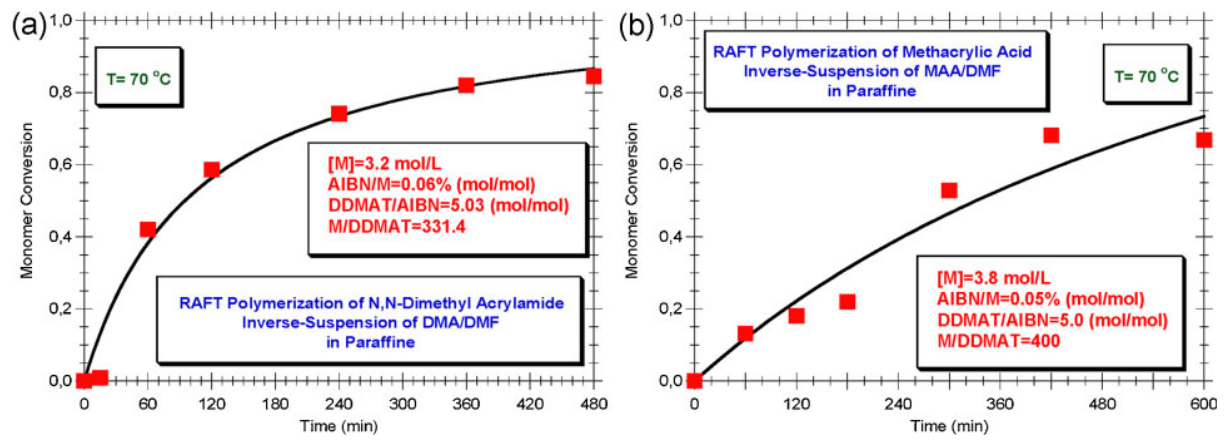

\section{Figure 8.}

Examples of observed dynamics of monomer conversion in different runs concerning the inverse-suspension RAFT synthesis of water compatible polymers and hydrogels (see Table 2). (a): DMA RAFT polymerization with $Y_{1}^{\text {RAFT }}=5.03$ (run 3). (b): MAA RAFT polymerization with $Y_{1}^{\text {RAFT }}=5.00$ (run 4).

observed with RAFT polymerization at $\mathrm{T}=70^{\circ} \mathrm{C}$ considering relatively high initial mole ratios RAFT agent/initiator (see Table 2). Kinetics of these RAFT polymerizations are illustrated in Figures 7-9 considering different water compatible monomers.

When RAFT network formation was sought using similar conditions (runs 6 to 8 in Table 2), lower gel fractions were observed in the products indicating a different crosslinking process when compared with FRP (theoretical and experimental kinetic studies on hydrogel formation by FRP where before reported $\left.{ }^{[20,21]}\right)$. Presence of such soluble phase is highlighted in Figure 10 (a) where the SEC traces of the final sample correspondent to run 6 in Table 2 are used to illustrate this issue. Note that besides the RI signal of the polymer (indicating the presence of soluble material), the LS signal is also presented and the coexistence of secondary population with low concentration but very high size can be observed. This secondary population was also observed in linear RAFT polymerizations, as below discussed, and is a possible consequence of non-ideal steps involved in the RAFT mechanism. Lower amounts of gel observed in these runs can result of the low primary chain length imposed by the RAFT process (mole ratio monomer/RAFT agent around 290). Dynamics of gel formation in RAFT
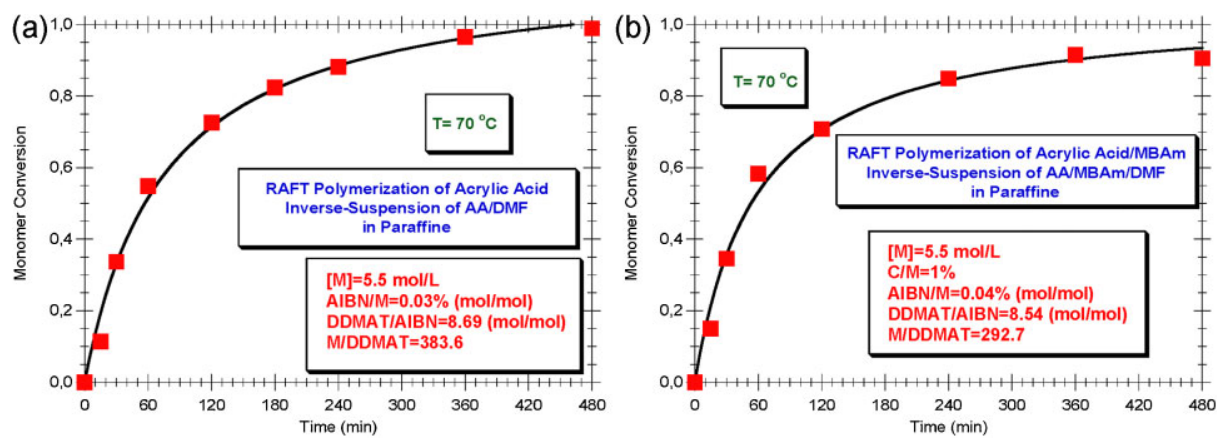

\section{Figure 9.}

Examples of observed dynamics of monomer conversion in different runs concerning the inverse-suspension RAFT synthesis of water compatible polymers and hydrogels (see Table 2). (a) AA RAFT polymerization with $Y_{I}^{\text {RAFT }}=8.69$ (run 5). (b) AA/MBAm RAFT polymerization with $Y_{1}^{\text {RAFT }}=8.54$ (run 6). 

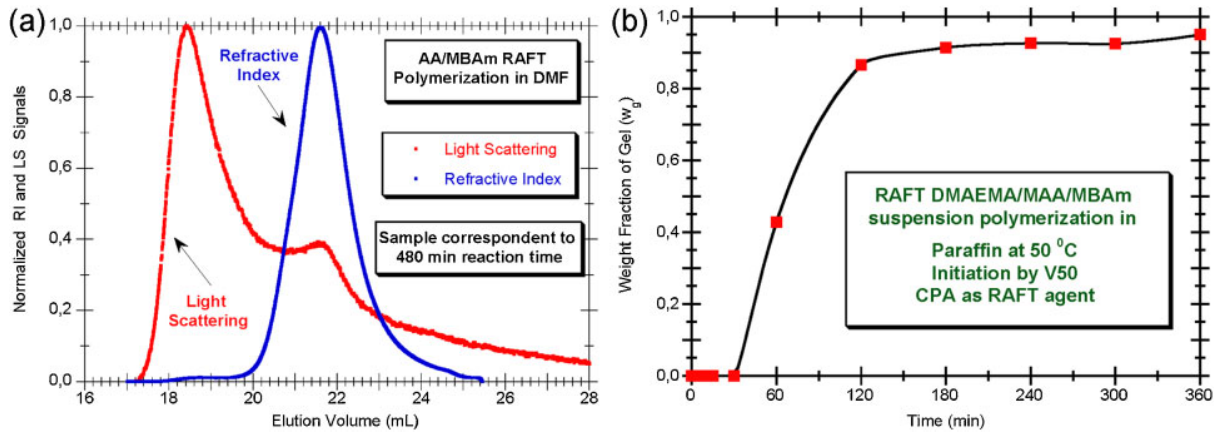

Figure 10.

(a) SEC chromatographic traces showing the presence of soluble material in AA/MBAm RAFT polymerization in DMF (run 6 in Table 2) and the formation of a secondary population with high molecular size and very low concentration. (b) Dynamics of gel formation during the RAFT synthesis of stimuli-responsive hydrogels. DMAEMA/MAA/MBAm RAFT polymerization at $50^{\circ} \mathrm{C}$ using CPA agent (run 4 in Table 3 ) is here considered for illustration purposes.

hydrogel synthesis is also illustrated in Figure 10 (b) considering different operation conditions, namely a lower mole ratio $Y_{I}^{R A F T}$ (see run 4 in Table 3 ), here with measurement of high final gel content. Mathematical modelling of RAFT nonlinear polymerization can be especially useful to aid with the interpretation of such results and to elucidate the mechanistic differences between RAFT and FRP crosslinking processes ${ }^{[19,31]}$.

Importance of multiple detection in the the SEC analysis of the RAFT products is also highlighted in Figure 11. Formation of a secondary population with very low concentration (almost negligible with RI or viscosity detection) but with very high molecular size (strong LS signal) was observed even with mono-vinyl monomer RAFT polymerization, as illustrated in Figure 11 (a). Dynamics of growth of such population is showed in Figure 11 (b). A possible justification for this phenomenon can be found in the framework of the RAFT slow fragmentation model leading to bimodal distributions formation. High concentration of intermediate radicals and the longer polymer chains associated to such species can be at the source of such nonideal behaviour, as recently showed using
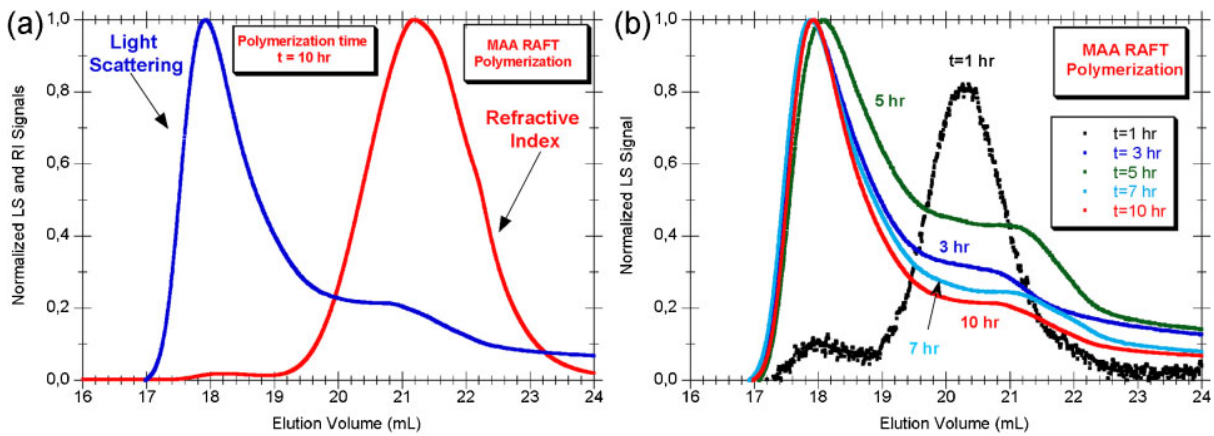

Figure 11.

(a) SEC chromatographic traces showing the formation of a secondary population with high molecular size and very low concentration in RAFT polymerization (data correspondent to run 4 in Table 2). (b) Dynamics of formation of such secondary population observed by light scattering in the same run. 
mathematical modelling ${ }^{[32]}$. Nevertheless, hydrolysis of the RAFT agent and/or polymerization both in aqueous and organic phases (with formation of controlled and non-controlled polymer populations) are also mechanisms with potential effect on the loss of control in these polymerization systems, as discussed above. Additional theoretical/experimental studies should help in the interpretation of the experimental observations here presented.

\section{Conclusion}

A comprehensive experimental program concerning the synthesis, characterization and testing (e.g. for drug delivery applications) of smart hydrogels was performed. Combining different aqueous compatible monomers, $\mathrm{pH}$ and temperature sensitive materials were obtained. Anionic, cationic and amphoteric network gel beads were produced using the inverse-suspension technique and the kinetics of their building process was studied by SEC with a tetra detection array and also using in-line FTIRATR. Comparison between the synthesis processes of these kinds of networks using FRP and RAFT polymerization (considering three different commercially available RAFT agents) could thus be experimentally performed. Important dissimilitudes in the dynamics of gel formation were identified when FRP mechanism is replaced by the RAFT polymerization. Some peculiarities of the RAFT synthesis were studied (e.g. solubility of the used RAFT agents in water and monomers considered, effect of initial composition/kinetics on gelation) in order to find proper conditions to produce the sought hydrogels using this CRP technique.

Only a limited information on the crosslinking process was possible to obtain with in-line FTIR-ATR monitoring, even using optimized conditions for IR measurements (bulk monomer polymerization in a dispersed media with low IR absorbance). Possibility of probe coating during the synthesis process and the low crosslinker content associated to hydrogels production are factors affecting negatively the use of such technique in this context. Use of offline FTIR analysis of isolated polymers $^{[23,31]}$, chemical analysis of pendant double bonds ${ }^{[15,23]}$ or ${ }^{13} \mathrm{C}$ labelling of the crosslinker $^{[24]}$ should provide improved information concerning the crosslinking process. Conversely, the use of multiple detection (especially including light scattering) in SEC analysis proved to be very important in order to obtain a rigorous characterization of products molecular architecture. In fact, the formation of an unexpected higher size secondary polymer population was detected by light scattering when RAFT polymerization is used, even with mono-vinyl monomers. These results can eventually be explained in the framework of recent theoretical findings describing the RAFT slow fragmentation model leading to bimodal distributions formation $^{[32]}$. Nevertheless, other mechanisms causing loss of control such as hydrolysis of the RAFT agent and/or simultaneous polymerization in aqueous and organic phases should also be considered in further developments on this research line.

Ongoing work in this research should hopefully also elucidate if really important gains in the sensitivity of these hydrogels can be attained replacing FRP by RAFT polymerization. Complementation of the experimental work here reported with new theoretical developments on the kinetic description of gels formation by the RAFT process $^{[31]}$ should also be explored to built up simulation tools aiding in the specification of synthesis conditions leading to tailored advanced materials.

Acknowledgments: Miguel Gonçalves acknowledges the financial support by FCT and FSE (Programa Operacional Potencial Humano/ POPH) through the PhD scholarship SFRH/ BD/76587/2011. Virgínia Pinto also acknowledges the financial support by FCT through researcher scholarship in the framework of the project PTDC/EQU-EQU/098150/2008 (Ministry of Science and Technology of Portugal/ Program COMPETE - QCA III/ and European 
Community/FEDER). This research has also been supported through the Marie Curie Initial Training Network "Nanopoly" (Project: ITN-GA-2009-238700) and by the program SAESCTN - PIIC\&DT/1/2011, Programa Operacional Regional do Norte (ON.2), contract NORTE-07-0124-FEDER-000014 (RL2_P3 Polymer Reaction Engineering).

[1] I. Galaev, B. Mattiasson, "Smart Polymers: Applications in Biotechnology Biomedicine", CRC Press, 2008.

[2] A. Bajpai, S. Shukla, R. Saini, A. Tiwari, "Stimuli Responsive Drug Delivery Systems: From Introduction to Application", i Smithers, 2010.

[3] Q. Yu, M. Zhou, Y. Ding, B. Jiang, S. Zhu, Polymer. 2007, 48, 7058.

[4] H. Gao, K. Min, K. Matyjaszewski, Macromolecules. 2007, 40, 7763 .

[5] H. Gao, W. Li, K. Matyjaszewski, Macromolecules. 2008, 41, 2335.

[6] M. A. D. Gonçalves, R. C. S. Dias, M. R. P. F. N. Costa, Macromol. Symp. 2010, 289, 1.

[7] M. A. D. Gonçalves, V. D. Pinto, R. C. S. Dias, M. R. P. F. N. Costa, Macromol. Symp. 2010, 296, 210.

[8] M. A. D. Gonçalves, R. C. S. Dias, M. R. P. F. N. Costa, Chem. Eng. Technol. 2010, 33, 1797.

[9] M. A. D. Gonçalves, I. M. R. Trigo, R. C. S. Dias, M. R. P. F. N. Costa, Macromol. Symp. 2010, 291-292, 239.

[10] N. Ide, T. Fukuda, Macromolecules. 1997, 30, 4268.

[11] S. Abrol, P. A. Kambouris, M. G. Looney, D. H. Solomon, Macromol. Rapid Commun. 1997, 18, 755.

[12] P. B. Zetterlund, M. N. Alam, H. Minami, M. Okubo, Macromol. F Rapid Commun. 2005, 26, 955.

[13] E. Tuinman, N. T. McManus, M. Roa-Luna, E. Vivaldo-Lima, L. M. F. Lona, A. Penlidis, J. Macromol. Sci. A. 2006, 43, 995.

[14] J. C. Hernándes-Ortiz, E. Vivaldo-Lima, A. Penlidis, Macromol. Theory Simul. 2012, 21, 302.

[15] M. A. D. Gonçalves, V. D. Pinto, R. C. S. Dias, M. R. P. F. N. Costa, L. G. Aguiar, R. Giudici, Macromol. React Eng. 2013, 7, 155.
[16] O. Yu, Y. Zhu, Y. Ding, S. Zhu, Macromol. Chem. Phys. 2008, 209, 551.

[17] O. Yu, S. Xu, H. Zhang, Y. Ding, S. Zhu, Polymer. 2009, 50, 3488.

[18] G. Jaramillo-Soto, E. Vivaldo-Lima, J. Aust. Chem. 2012, 65, 1177.

[19] D. Wang, X. Li, W. J. Wang, X. Gong, B. G. Li, S. Zhu, Macromolecules. 2012, 45, 28.

[20] M. A. D. Gonçalves, V. D. Pinto, R. C. S. Dias, M. R. P. F. N. Costa, Journal of Nanostructured Polymers Nanocomposites. 2013, 9, 40.

[21] M. A. D. Gonçalves, V. D. Pinto, R. C. S. Dias, M. R. P. F. N. Costa, Macromol. Symp. 2011, 306-307, 107. [22] S. Salehpour, M. A. Dubé, Macromol. React. Eng. 2012, 6, 85.

[23] M. Hecker, "Experimentelle Untersuchungen und Monte-Carlo-Simulation netzwerkbildender Copolymerisationen". Fortschritte der Polymerisationstechnik. II (H.U. Moritz ed.) Wissenschaft Technik Verlag Berlin, ISBN 3-89685-353-8, 2000.

[24] D. J. Arriola, S. S. Cutié, D. E. Henton, C. Powell, P. B. Smith, J. Appl. Polym. Sci. 1997, 63, 439.

[25] I. Chaduc, M. Lansalot, F. D'Agosto, B. Charleux, Macromolecules. 2012, 45, 1241.

[26] L. Ouyang, L. Wang, F. J. Schork, Macromol. React. Eng. 2011, 5, 163.

[27] G. Qi, C. W. Jones, F. J. Schork, Macromol. Rapid. Commun. 2007, 28, 1010.

[28] W. Zhang, B. Charleux, P. Cassagnau, Macromolecules. 2012, 45, 5273.

[29] (a) W. Zhang, F. D'Agosto, O. Boyron, J. Rieger,

B. Charleux, Macromolecules. 2012, 45, 4075. (b)

I. Chaduc, M. Girod, R. Antoine, B. Charleux, F. D'Agosto, M. Lansalot, Macromolecules. 2012, 45, 5881.

[30] W. Zhang, F. D’Agosto, P. Dugas, J. Rieger, B. Charleux, Macromolecules. 2013, 54, 2011.

[31] M. A. D. Gonçalves, V. D. Pinto, R. C. S. Dias, M. R. P. F. N. Costa, J. C. Hernándes-Ortiz, Modeling Studies on RAFT copolymerization of Styrene/Divinylbenzene in Aqueous Suspension, Presented at the 11th Workshop on Polymer Reaction Engineering, Hamburg, 2013.

[32] I. Zapata-González, E. Saldívar-Guerra, J. OrtizCisneros, Macromol. Theory Simul. 2011, 20, 370. 